This is the final peer-reviewed accepted manuscript of:

L. Mazzocchetti, T. Benelli, E. Maccaferri, S. Merighi, J. Belcari, A. Zucchelli, L. Giorgini "Poly-m-Aramid Electrospun Nanofibrous Mats as High-Performance Flame Retardants for Carbon Fiber Reinforced Composites" Composites: Part B, 145, 252260 (2018).

The final published version is available online at: https://doi.org/10.1016/j.compositesb.2018.03.036

Rights / License:

The terms and conditions for the reuse of this version of the manuscript are specified in the publishing policy. For all terms of use and more information see the publisher's website.

This item was downloaded from IRIS Università di Bologna (https://cris.unibo.it/)

When citing, please refer to the published version. 


\section{Accepted Manuscript}

Poly-m-aramid electrospun nanofibrous mats as high-performance flame retardants for carbon fiber reinforced composites

Laura Mazzocchetti, Tiziana Benelli, Emanuele Maccaferri, Stefano Merighi, Juri Belcari, Andrea Zucchelli, Loris Giorgini

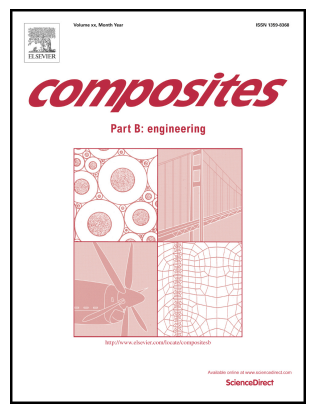

PII: S1359-8368(18)30279-8

DOI: 10.1016/j.compositesb.2018.03.036

Reference: JCOMB 5595

To appear in: Composites Part B

Received Date: 23 January 2018

Revised Date: 1 March 2018

Accepted Date: 20 March 2018

Please cite this article as: Mazzocchetti L, Benelli T, Maccaferri E, Merighi S, Belcari J, Zucchelli A, Giorgini L, Poly-m-aramid electrospun nanofibrous mats as high-performance flame retardants for carbon fiber reinforced composites, Composites Part B (2018), doi: 10.1016/j.compositesb.2018.03.036.

This is a PDF file of an unedited manuscript that has been accepted for publication. As a service to our customers we are providing this early version of the manuscript. The manuscript will undergo copyediting, typesetting, and review of the resulting proof before it is published in its final form. Please note that during the production process errors may be discovered which could affect the content, and all legal disclaimers that apply to the journal pertain. 

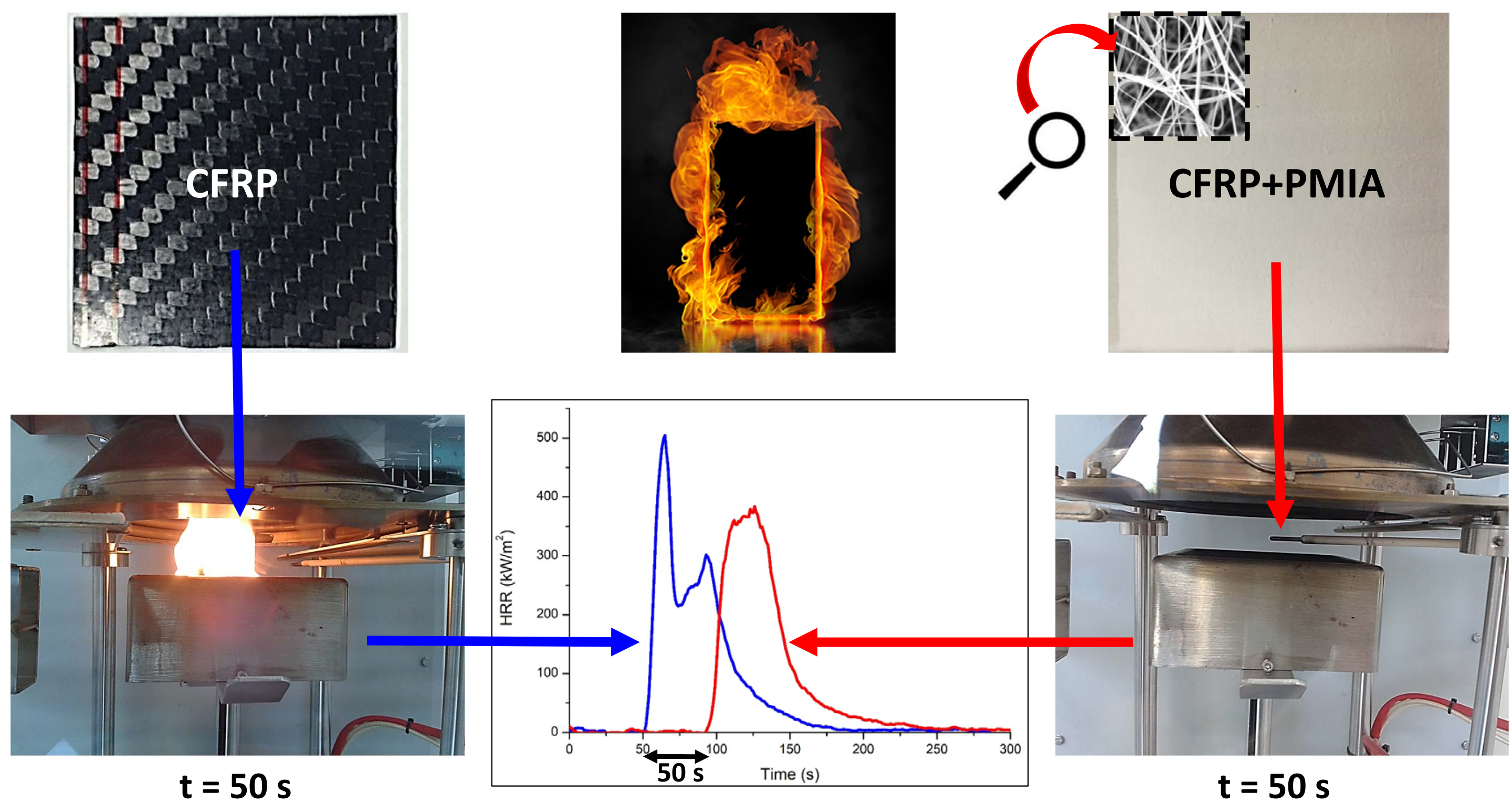

$\mathbf{t}=\mathbf{5 0} \mathrm{s}$ 


\title{
Poly-m-Aramid Electrospun Nanofibrous Mats as High-Performance Flame Retardants for Carbon Fiber Reinforced Composites
}

Laura Mazzocchetti $^{\mathrm{a}, \mathrm{b}}$, Tiziana Benelli ${ }^{\mathrm{a}, \mathrm{b}}$, Emanuele Maccaferri ${ }^{\mathrm{a}}$, Stefano Merighi ${ }^{\mathrm{a}, \mathrm{b}}$, Juri Belcari ${ }^{\mathrm{b}, \mathrm{c}}$, Andrea Zucchelli ${ }^{\mathrm{b}, \mathrm{c}}$, Loris Giorgini ${ }^{\mathrm{a}, \mathrm{b}}$

a Department of Industrial Chemistry “Toso Montanari”, University of Bologna, Viale Risorgimento 4, 40136 Bologna, Italy.

${ }^{b}$ Interdepartmental Center for Industrial Research on Advanced Applications in Mechanical Engineering and Materials Technology, CIRI-MAM, University of Bologna, Viale Risorgimento 2, 40136 Bologna, Italy.

${ }^{c}$ Department of Industrial Engineering, University of Bologna, Viale Risorgimento 2, 40136 Bologna, Italy;

\begin{abstract}
For the first time an innovative approach to manufacture flame shield coatings by producing thin polyaramidic nanofibrous sheets via electrospinning is presented. Nanofibrous mats with a well-defined alignment provide a thin, compact but pliable layer of intrinsically flame-retardant material. The electrospun membrane (about $0.4 \mathrm{~g} / 100 \mathrm{~cm}^{2}$ ) displays good mechanical properties and easy handling and could be applied onto epoxy-carbon fiber composites to modify their flame behaviour. Conecalorimetric tests show time to ignition doubled for the coated material and a dramatically reduced ability to propagate fire once ignited. Finally, addition of a simple polyaramidic nanofibrous membrane is proved able to change the flame behaviour of a composite from a high-risk material class, to an intermediate one.
\end{abstract}

Keywords.

A. Aramid fibre; A. Nano-structures; A. Polymer-matrix composites (PMCs); B. High-temperature properties; 


\section{Introduction}

Structural materials are widely used in a broad range of fields like building, furnishing, aeronautics, automotive, and recently the request for lightweight high performance structural materials pushed the market from iron, clay bricks, stones, steel and reinforced concrete to more suitable products, in particular polymers and polymer composites [1;2]. Polymers and polymeric composites find application in a wide range of fields and their production is continuously growing, owing to their overall cheapness and the possibility to produce materials with very specific mechanical, thermal, and chemical properties associated to low density. Among all the positive aspects of polymer matrix composites, a relevant limit to their application is the flammability issue. The polymeric, i.e. organic, matrix already decomposes at moderate temperatures, below $300-400^{\circ} \mathrm{C}$, with heat evolution associated to the production of smoke, soot and often also toxic volatiles [3]. The global annual burden associated to fire accidents ranks around tens of billions (\$), a figure covering roughly $1 \%$ of global annual Gross Domestic Product (GDP). Beside the material damages, data referring to just the European countries report thousands of fire related deaths, together with an even higher number of casualties, sometimes suffering for life-long invalidating injuries [4]. With the aim to stop, or at least delay, the thermal decomposition and combustion processes, Flame Retardant systems (FRs) are often used when ignitable materials, such as polymer composites, are involved. Fire retardancy, as an outstanding element of safety, is one of the key challenges pursued in the present days industry.

In order to achieve a better fire behaviour for polymeric composites, different routes can be approached [5], such as (A) the use of an intrinsic flame resistant polymer, (B) the addition of FRs to the bulk of the materials, or (C) the application of coatings with a flame retardant and/or intrinsic flame resistant materials. The first approach implies the choice of a specific macromolecular system, leading to a completely new product and product design, which, when available, is usually associated with long development times and a cost increase. The second approach, instead, requires the use of specifically formulated FRs additives, which can be based on different principles. Among the most renown FRs are halogenated compounds, which dates their applications back to the thirties and are now addressed as possible sources of toxic compounds [6], recently causing their ban due to the adverse environmental effects [7]. Hence inorganic hydrates, borates, silicates, carbonates; metal oxides, sulphides, phosphorus, silicon and nitrogen compounds have been actively pursued as alternative FRs [8]. Owing to the relatively high percentage of additive required, such polymeric formulations might suffer for a drop in the mechanical performances. Since the ability of nanofillers of different nature is well renown to 
boost the mechanical properties of polymers [9-11], the possibility of producing nanofillers, such as clays [12], organoclays [13], or graphenic derivatives [14-16] conjugating the nanosized dimension and the flame retardant ability might help in promoting the mechanical properties as well as fire behaviour of the modified polymer. This approach, however, requires the homogeneous modification of the whole bulk mass of the material, while it has been recognized the paramount role played by the polymer surface during combustion since the heat produced and the combustible gases evolved because of the thermal degradation, both promoting the combustion, occur at the surface [17]. Superficial coatings, thus, may allow to retain the mechanical behaviour of the pristine bulk material, while contemporary regulating the combustion process at the outer boundary[18]. Furthermore, they can be applied to semi-finished or pre-existing objects which cannot be easily bulk modified. Several examples of coatings used to add flame retardant properties to a wide spread of materials are reported in literature, such as intumescent paints, which create an insulating foam reducing the spreading of flame and preventing oxygen penetration, are commonly used [19]. Other reported applications are represented by coating with carbon nanofiber based nanopaper [20], deposition of inorganic nanosheets [21], and graphene oxide [22]. A new way to perform fire resistant surface modification could be the application of polymeric nanofibrous mats onto the outer boundary of the inflammable material. In this context, polyaramids, in spite of their organic nature, are a class of polymers that exhibits remarkable flameproof properties [23]. The most representative polyaramids are the meta-aramid poly(meta-phenylene isophtalamide) - PMIA (NOMEX, DuPont) and the para-aramid poly(para-phenylene terephtalamide) - PPTA (KEVLAR, DuPont), the former being well known for its good thermal resistance [24], the latter for its excellent mechanical and chemical properties, in addition to thermal resistance [25]. The drawback in PPTA application is the difficulty to process the polymer because it only dissolves in concentrated sulphuric acid and at a relatively low concentration. PMIA, on the contrary, is an inherently flame resistant and self-extinguishing polymer easier to process. In a recent work, PMIA fabrics have been tested as FRs in polyurethane foam s[26], finding that the number of aramidic layers strongly affects the fire behaviour of the substrate. A significant improvement of the Time To Ignition (TTI), compared to the neat foam, was achieved when a minimum of four fabric layers were used. However, there is a relevant increase of both weight and thickness of the final product when more fabric layers are used. Coating layers constituted by nanofibrous PMIA could thus be a viable way to improve fire resistance without significant weight nor thickness increase. Nowadays commercial nanofibrous PMIA, Nomex Nano - DuPont, are used mainly to improve smoke shielding properties of PMIA-based protective clothing [27]. 
Differently from PPTA, PMIA can be dissolved in amidic solvents, in the presence of inorganic salts, and the solution can be easily processed via electrospinning to produce nanofibrous mats [28]. The aim of the present paper is thus to present, for the first time, an innovative approach to flame shield coatings via thin PMIA electrospun nanofibrous sheets. The nanofibrous mats will be optimized and fully characterized and their fire retardancy ability will be tested upon application onto the surface of Carbon Fiber Reinforced Polymer (CFRP) panels.

\section{Material and Method}

\subsection{Materials}

Poly (m-phenylene isophtalamide) (PMIA) and lithium chloride ( $\mathrm{LiCl})$ (Sigma-Aldrich) were dried before use. They were treated in oven at $110^{\circ} \mathrm{C}$ for 3 and 24 hours respectively. N,N-dimethylacetamide (DMAc) (SigmaAldrich) was treated according to previously reported procedures [29]. The distillation was performed at $64^{\circ} \mathrm{C}$ and $18 \mathrm{mmHg}$ under nitrogen flow. After distillation, the solvent was stored under nitrogen over molecular sieves ( 4 Å) before use. CFRP panels obtained by carbon fiber-epoxy resin prepregs were kindly supplied and cured by Grafite Compositi - Castel San Pietro Terme (BO) Italy. The paint used is a polyurethane PPG Deltron D800/D841 (2/1 vol.) system. All other materials were used as received without further purification.

\subsection{Nanofibrous mats production}

Polymeric solutions were prepared dissolving $\mathrm{LiCl}$ in DMAc under magnetic stirring and mild warming $\left(40^{\circ} \mathrm{C}\right)$ for at least 2 hours, then PMIA was added and the system stirred at $70^{\circ} \mathrm{C}$ until formation of homogeneous solutions. The investigated electrospun solutions and their weight percent composition are reported in Table 1. PMIA nanofibrous mats were produced via electrospinning technique using a Spinbow® electrospinning machine equipped with four $5 \mathrm{~mL}$ syringes connected to needles $(0.84 \mathrm{~mm}$ internal diameter) with Teflon tubing. Fibers were collected on a rotating drum $(60 \mathrm{rpm})$ covered with polyethylene $(\mathrm{PE})$ coated paper. The actual processing parameters are reported in Table 1. After process parameter optimization, and collecting fibers for 40 hours, 30x40 cm electrospun mats with an average $80 \mu \mathrm{m}$ thickness were obtained. Nanofibrous mats were finally washed with distilled water and dried at $70^{\circ} \mathrm{C}$ in order to remove $\mathrm{LiCl}$ salt residue, as previously reported $[28 ; 30]$.

\subsection{CFRP samples preparation for fire resistance tests}

Two kinds of sample were prepared with CFRP panels and washed and dried electrospun PMIA mats (PMIA w) produced with the PMIA 14-3,5 solution. In the first case mats were applied onto the last layer of prepreg of 
the CFRP (10x10 cm, 1,5 mm thick) before the curing process in autoclave (Figure 1A, 1B, 1C). In the second kind of sample an adhesive paint interlayer was used to apply a PMIA nanofibrous mat onto the surface of a cured CFRP panel. In particular, 10x10 cm CFRP specimens (Figure 1D) 1,38 mm thick, were covered on both sides with a paint layer (50 $\mu$ m thickness) (Figure 1E) and then PMIA mats were applied on top in order to completely cover the edges (Figure 1F). Samples were pressed with a roller in order to promote adhesion, then they were dried at $70^{\circ} \mathrm{C}$ for 20 minutes. Hence CFRP with incorporated PMIA (hereafter defined as C-PMIA inc W) and CFRP with PMIA nanofibers applied superficially with paint (hereafter defined as CP-PMIA W) were prepared while CFRP specimens (hereafter defined as C-Neat) and CFRP coated with the paint (hereafter defined as CP-Neat) were produced and taken as reference to compare with materials containing PMIA.

\subsection{Characterization}

In order to investigate the morphological aspect of the obtained nanofibers, micrographs were taken with a Scanning Electron Microscope (SEM) Phenom ProX. Energy dispersive X-rays analysis (EDX, Phenom ProX) was used to confirm the absence of chloride ions in nanofibrous membranes after the washing treatment. Thermogravimetric analyses (TGA) were carried out on a TA Instruments SDT Q600, in air atmosphere (flow rate: $100 \mathrm{ml} / \mathrm{min}$ ) heating from $25^{\circ} \mathrm{C}$ to $600^{\circ} \mathrm{C}$ at $10^{\circ} \mathrm{C} / \mathrm{min}$, followed by 30 minutes isotherm. Differential Scanning Calorimetry (DSC) measurements were carried out on a TA Instruments DSC Q2000 apparatus equipped with RCS cooling system, heating twice $3-5 \mathrm{mg}$ samples in aluminium pans from $0^{\circ} \mathrm{C}$ to $350^{\circ} \mathrm{C}$ at $10^{\circ} \mathrm{C} / \mathrm{min}$, with intermediate cooling run at $-10^{\circ} \mathrm{C} / \mathrm{min}$. Stress- strain tests were performed using a Instron 5960 Series Dual column table frames. Flame behavior studies were performed using an oxygen consumption calorimeter (Fire Testing Technology Limited FFT Cone Calorimeter). The tests were performed at an incident Heat flux of $35 \mathrm{~kW} / \mathrm{m} 2$ in horizontal orientation using the cone shaped heater and specimens of $100 \times 100 \mathrm{~mm}^{2}$. Three samples per formulation were tested. The Cone Calorimeter provides the following parameters: Heat Release Rate (HRR), calculated from the oxygen consumption, Time To Ignition (TTI), Time of Flame out (TOF), Average Heat Release Rate (HRR as a function of time), peak of Heat Release Rate (pHRR), Total Heat Release (THR) and Time To pHRR (TTP). Experimental data were reproducible within $\pm 10 \%$. The Average Heat Release Rate is correlated to the heat released in a room where the flammable materials are not all ignited at the same time. Two other parameters were calculated from the measured cone-calorimeter data as they have been accepted as representative of the polymer combustion behavior in a real fire [31;32]: Fire Performance Index (FPI), calculated as pHRR/TTI, and Fire Growth Rate Index (FIGRA) calculated as pHRR/TTP. 


\section{Results and discussion}

\subsection{PMIA nanofibrous mats production}

It is well renown that the electrospinning of a polymer requires either it being in the melt or in solution. The latter accounts for the easiest process, provided that the solution displays suitable features in terms of molecular weight and concentration of the macromolecular solute of choice, and convenient solvent, solution viscosity and conductivity. Owing to the poor solubility of PMIA in many common solvents, the possibility of electrospinning might be significantly hampered, since solvent candidates providing full polymer solubilisation and contemporary displaying properties suitable for the process are limited [33]. Previous attempts at solubilizing the meta-aramidic polymer highlighted that the contribution of some salts is required to help disrupt the tight macromolecular packing based on intra- and inter-molecular hydrogen bonding characterizing the solid state of PMIA. The solvent system DMAc/LiCl represents, by far, the best available choice [28;30;33], since it allows obtaining not only a homogeneous solution, but the mixture also displays convenient parameters to be processed via electrospinning. $\mathrm{LiCl}$ is, indeed, fundamental for the polymer dissolution because in $\mathrm{DMAc} \mathrm{Li}^{+}$cations coordinate the carbonyl oxygen of DMAc, forming a complex, while $\mathrm{Cl}^{-}$anions break the inter-chain hydrogen bonds, allowing the solubilization [33]. Moreover, it contributes to the solution conductivity, making the overall mixture suitable for the electrospinning process.

With the aim of optimizing the system for the electrospinning process, different combinations of polymer (6$18 \%$ wt) and salt (1-8\%wt) concentrations in DMAc were investigated. Polymer concentration, indeed, strongly affects the solution viscosity, one of the most important solution parameter that influences electrospinning process and nanofibers morphology. Moreover, an increase in salt concentration heavily raises not only the solution viscosity, but also the conductivity, another fundamental requirement to obtain an electrospinnable system. Solutions made of $14 \%$ wt of PMIA and 2 to $3.5 \%$ wt $\mathrm{LiCl}$ in DMAc were found to be the best choice for nanofibrous membrane production, leading to a stable electrospinning process (no occlusion of needles). The nanofibers obtained show small and homogeneous diameters (205-220 for unwashed and 171-197 nm for washed membrane) with no beads nor other defects. After the electrospinning process, assuming that all the solvent evaporates during the procedure, the obtained nanofibrous mat would still contain all the added $\mathrm{LiCl}$, making it up to $12.5-20 \%$ wt of salt in the polymeric membrane.

With the aim of evaluating the effect of the $\mathrm{LiCl}$ presence on the nanofibrous mats properties, the produced membranes were washed with distilled water and dried at $70^{\circ} \mathrm{C}$ overnight [34].In Figure 2 SEM micrographs 
of washed (PMIA W) and unwashed mats (PMIA UW), produced from a PMIA 14-3,5 solution, are reported showing a decrease in the average fibers diameter from 220 to $197 \mu \mathrm{m}$ (10.5\% reduction) upon salt removal. The cleaning action, however, does not lead to macroscopic deformation, nor shrinkage.

The removal of the $\mathrm{LiCl}$ salt was confirmed by elemental analysis EDX, which show the disappearance of the signal ascribed to chloride ion after washing (Figure 3). It is worth noting that environmental parameters, Temperature and Humidity, considerably affect the morphology of the obtained nanofibers, resulting in an overall random arrangement when the relative humidity is maintained around $20 \%$ or in prevalently aligned fibers if $\mathrm{RH}$ raises above $45 \%$ (within a temperature range of $25-30^{\circ} \mathrm{C}$, see Figure 4). The mat obtained from the solution containing $2 \%$ of $\mathrm{LiCl}$ (Figure 4A) was produced with a relative humidity of $45 \%$ and the fibers are clearly preferentially aligned. On the contrary both mats obtained from solution containing $2,5-3,5 \%$ of $\mathrm{LiCl}$ (respectively Figure 4B and C) were produced with a 26\% RH and it is possible to observe that in both mats nanofibers seem to be randomly oriented. Hence, depending on the final use, the environmental conditions should be finely tuned to tailor the nanofibrous membrane morphology. Mats made of nanofibers with a high degree of orientation were used in the present work, since it was considered that a prevailing orientation direction would provide a higher density of fibers per volume unit than the case of a completely random arrangement, and the higher the number of fibers per volume, the better should be the fire resistance.

\subsection{Thermal characterization}

The obtained nanofibrous mats were thermally characterized by TGA and DSC analysis. In Figure 5 the thermogravimetric curves, carried out heating in air from RT to $600{ }^{\circ} \mathrm{C}$, of neat PMIA, PMIA W and PMIA

UW are reported. Pristine commercial PMIA shows a first weight loss starting around $80^{\circ} \mathrm{C}$, which can be ascribed to the water absorbed on the material as proved by the on-line FT-IR analysis of the evolved gas. Such a behaviour is typical of polyamidic/polyaramidic materials and correspond to an average water content around $6 \%$ wt. The massive PMIA degradation occurs above $400^{\circ} \mathrm{C}\left(408^{\circ} \mathrm{C}\right.$ onset $)$ and no residue is observed.

As expected, the adsorbed water fraction strongly increases in the nanofibrous mat containing LiCl (PMIA UW), owing to the strong hygroscopic nature of the inorganic compound. It is worth noting that the degradation rate of the $\mathrm{LiCl}$ containing nanofibers is lower than neat-PMIA and the TGA solid residue at $600^{\circ} \mathrm{C}$ is more than twice the expected value $(20 \%$ of $\mathrm{LiCl})$ (if the released water fraction is not considered in the total starting weight). Such a behaviour suggests that the applied temperature program is not able to lead to a full degradation of the organic fraction, thus the $\mathrm{LiCl}$ seems to act as a thermal stabilizer. This is confirmed 
by PMIA W (Figure $5 \mathrm{c}$,) which, upon removal of the inorganic component, goes back to a behaviour similar to that one of pristine PMIA, with a comparable water content (about 6\%) and a full degradation within the same temperature range.

In Figure 6 the DSC curves of pristine PMIA, PMIA UW and PMIA W in first and second heating scans are reported. In the first scan all the samples display an endothermic transition between 50 and $150^{\circ} \mathrm{C}$ which, upon the discussion of the TGA analysis, can be ascribed to the evaporation of the water absorbed by nanofibers. It is worth noting that in PMIA UW first scan thermogram (Figure 6A, b) this transition is more intense and with the maximum of the peak shifted to higher temperature than neat and PMIA W. Such a behaviour agrees with TGA analysis and stems from the presence of $\mathrm{LiCl}$ which, being very hygroscopic, strongly increases the absorbed water fraction. The small modification of water content between pristine PMIA and washed PMIA W can instead be explained by the higher superficial area of nanofibers, which allows a slightly higher water fraction to be adsorbed. In Table 2 the temperatures and relative $\Delta \mathrm{H}$ of these endothermic DSC transitions registered in the first DSC scan are reported. As expected, in the second scan (Figure 6B) no endothermic transitions are detected, having water been completely removed. Upon water removal (in second scan), neat PMIA displays a $T_{\mathrm{g}}$ centred at $304^{\circ} \mathrm{C}$, this value increasing for the PMIA UW. Such a behaviour could be triggered by stronger intermolecular interactions between the very stiff polyaramidic macromolecules and the salt, when water molecules are no more present. On the other hand, the decrease in $T_{\mathrm{g}}$ displayed by PMIA W can be explained by the increase of free volume between nanofibers after the washing process, which is not compensated anymore by the polymer salt interaction, as in the case of PMIA UW.

\subsection{Mechanical characterization}

In order to better understand the mechanical properties of the investigated PMIA mats, specimens were analysed with stress-strain tests in tensile mode. Samples were produced from solution of different salt content (PMIA 14-2 and PMIA 14-3,5, as described in Table 1) and were studied both washed (W) and unwashed (UW) nanofibers, collected along the direction corresponding either to the longitudinal (//) or the transverse $(\perp)$ direction of the rotating drum collector as reported in Figure 7. It is worth to point out that such mechanical test provides an indication of the mechanical behaviour of the nanofibrous object, which is mainly composed of voids. As a matter of fact, while the intrinsic material properties play a role in the definition of the nanofibrous mat behaviour upon mechanical deformation, the overall response is much more affected by the nanofibers morphology, tridimensional assembly (easiness of macroscopic fibers disentanglements), membrane thickness 
and salt presence. Thus, the electrospinning settings, membrane properties as well as the testing condition may strongly affect the final results. As shown in Table 3 both PMIA 14-2 and PMIA 14-3,5 washed nanofibrous mats displayed a higher Young's modulus along the longitudinal direction than along the transverse one. In particular, the best results were achieved for washed and longitudinally oriented PMIA 14-2 W // nanofibers. All the results showed are in accordance to the work of Su-Yeol Ryu [34] where the best mechanical properties where obtained for the washed mats oriented along the longitudinal direction of the rotating drum. As expected [34], all the sample of the unwashed specimens showed very low Young's module also for fibers collected in longitudinal directions, as reported for PMIA 14-2 UW // as an example. This behaviour can be ascribed to the presence of $\mathrm{LiCl}$, which, beside interacting at the macromolecular level with the polyaramid, brings in the mat a high water fraction which could favour the fibers disentanglements when subject to tensile deformation, even along the fiber's prevailing orientation direction. While, at a first sight, the fibers alignment in the nanofibrous membrane appear different (see Figure 4), namely PMIA 14-2 looks aligned while PMIA 14-3.5 seems random, the previously discussed results do not seem consistent with such a visual observation. With the aim of solving this issue, is was thus defined a so-called Alignment Index $\left(\mathrm{I}_{\mathrm{a}}\right)$, applied to a membrane produced by the same solution and measured along two perpendicular directions, as reported in Eq 1:

$I_{a}=\frac{\text { Young's modulus } / /}{\text { Young's modulus } \perp}$

where $I_{a}$ is the ratio between the Young's modulus of nanofibers collected along longitudinal directions and the Young's modulus of nanofibers collected along transverse directions of the rotating drum collector. This parameter provides a numerical evaluation of the fibers orientation, far more significant than the visual assessment carried out via SEM micrographs that solely refers to the outer portion of fibers. In particular, the higher is the extent of fibers aligned along a preferential direction, the greater the $\mathrm{I}_{\mathrm{a}}$ value will be. The highest degree of alignment, $I_{a}$, was obtained for PMIA 14-2 samples; worth of noting is also the relevant $I_{a}$ of the PMIA 14-3,5 mat, suggesting that a significant fibers alignment can be achieved also with this solution.

\subsection{Testinf of Fire Behaviour}

Being the PMIA fire resistant behaviour well established [24;26], the effect that its application in the form of a micrometric thick superficial layer of nanofibers might induce on the flame behaviour of a carbon fiber reinforced composite (CFRP) has been investigated. In particular, we used washed mats produced with a PMIA 14-3,5 solution. The batch PMIA 14-3,5 displays a lower fibers alignment, but it was chosen for fire testing 
behaviour instead of PMIA 14-2 since with this formulation it was possible to produce a stable electrospinning process that lead to nanofibrous mats without macroscopic defects even after 50 hours. During the 50 hours spinning of the PMIA 14-2 solution, in fact, process issues were reported and it was not possible to produce a flawless membrane suitable for future tests. In this work, PMIA nanofibrous mats were applied onto the surface of a CRFP panel (C-Neat) in two different modes. In the first case the PMIA mat was incorporated in the last layer of a CFRP panel (C-PMIA inc W) before the curing process of the composite material (Figure 1A-C). It is a simple way to add a superficial membrane layer to a composite material since there are no product post treatments and, after the cure, the adhesion between the CFRP and the nanofibrous mat is perfect, and after the curing treatment it is possible to see the nanofibrous mat completely incorporated under the epoxy resin layer of the CFRP panel (Figure 1C). In second case, the PMIA membrane was applied onto the surface of a cured CFRP specimen using a paint layer as adhesive (sample (CP)-Neat and CP-PMIA W). In this case too, the application of the PMIA mat is simple, since a final paint layer is always applied onto CFRP as a finishing to protect the composite material. Then, the flame behaviour of the obtained samples was assessed by conecalorimeter, which is one of the most effective bench-scale methods for studying the flammability properties of materials[35]. Tests were carried out with a heat flow of $35 \mathrm{~kW} / \mathrm{m}^{2}$ (about $650^{\circ} \mathrm{C}$ ) which can be roughly compared to a small-scale fire. The instrument can work with a heat flow ranging between 5 and $100 \mathrm{~kW} / \mathrm{m}^{2}$, the higher irradiation levels give better reproducibility, more clearly defined ignition, and shorter measurement times, but correspond to more fully developed fires. Often a smaller irradiation level better fits to the fire protection goals, since it accounts for the material behaviour in a condition close to the starting of a fire, showing its ability to ignite and propagate. The main data monitored during the test are: Heat Release Rate (HRR), reported in $\mathrm{kW} / \mathrm{m}^{2}$ which represents the irradiated thermic power released by the sample per square meter; Total Heat Release (THR), reported in $\mathrm{MJ} / \mathrm{m}^{2}$ which is the total heat released by the sample, from ignition to extinction; Time to ignition (TTI), reported in seconds it represents the time span between the start of the sample exposition to the heating source, in the presence of an ignition system, and the moment when the first flame is detected. Moreover, two additional parameters were calculated, starting from the measured values. First, the Fire Performance Index (FPI), reported in $\mathrm{kW} / \mathrm{m}^{2} \mathrm{~s}$, providing an overall assessment of the fire safety of a material in the cone calorimeter test, has been calculated as the ratio between the maximum value of the Heat Release Rate (pHRR) and the Time To Ignition (TTI): FPI is related to the time required for flashover. Then Fire Growth Rate Index (FIGRA), reported in $\mathrm{kW} / \mathrm{m}^{2} \mathrm{~s}$, a parameter helpful in ranking the materials in 
terms of potential fire safety because it combines the most intense heat release (pHRR) and time to achieve it (Time To Peak, TTP). FIGRA is a good descriptor of the burning behaviour of building products. The complete set of data obtained is reported in Table 4 and is based on the average of three runs for each sample, while in

Figure 8 and Figure 9 cone-calorimeter curves significant of the batch behaviour are reported. In Figure 8 the HRR and THR curves for C-PMIA inc W are compared with the plain reference of CFRP with no PMIA incorporated layer (C-Neat), sampling the same carbon fiber epoxy resin composite used to prepare the modified sample. The data reported indicate no significant effect of the nanofibrous mat presence. The lack of effect of the incorporated PMIA membrane can be due to the presence of the epoxy resin external layer which burn before the nanofibrous mat can provide its action. For the sake of comparison, in Figure 9 HRR and THR curves for CP PMIA W are compared with the plain reference with no PMIA coating layer, sampling the same carbon fiber epoxy resin composite used to prepare CP PMIA W samples, covered with the same painting used as adhesive for the nanofibers (sample CP Neat). The data reported show a remarkable effect of the nanofibrous mat presence concerning, in particular, the time to ignition (TTI) value: the samples covered with PMIA nanofibers need longer time to ignite compared to the CFRP neat samples. The ignition delay observed for the samples covered with nanofibers was approximately 50 seconds if compared with the samples CP Neat, thus TTI is more than doubled. This result is really promising since the delay was obtained using just a small amount of nanofibrous material $\left(0.24-0.27 \mathrm{~g} / 100 \mathrm{~cm}^{2}\right)$. In a real fire scenario the escape-time plays a fundamental role in the survival of the people involved and therefore the longer the ignition time, the better the material. Moreover, there is also a significant pHRR decrease upon application of a thin nanofibrous polyaramidic outer layer, though not quite as intense as for the TTI value. Overall, however, the THR values obtained for C-Neat, C-PMIA inc W, CP Neat and CP PMIA W are comparable, as shown in Figure 9 A-B and Table 4. Such a trend is easily summarized in Figure 10, where some among the most significant cone calorimeter data, are reported as percent variation of the PMIA covered sample with respect to the plain one (taken as $100 \%$ reference value), in order to better highlight substantial differences. The value reported in Figure 10 are particularly relevant because, according to the work of Petrella [36], they can be combined to provide some indication for the ability of the investigated material to propagate the fire. While the ratio of pHRR/TTI has been defined as Fire Performance Index, this index has been recognized also as an indication of the propensity of the material to flashover [36]. The flashover is a possible consequence of a fire ignition, which require specific conditions to occur, such as an intense and instantaneous amount of heat (high pHRR, 
short TTI) and is obviously an additional threat in the case of fire. Correlation of THR and FPI values leads to a good assessment of the material tendency to propagate a fire; hence, while in the present case THR is almost unaffected, FPI being more than halved thank to the PMIA superficial nanofibers accounts for a significant higher safety of CP PMIA W. According to the work of Petrella [36], a classification can be issued based on the FPI index, with the following indication:

$\begin{array}{ll}\mathbf{0 . 1}<\text { FPI }<1 & \text { Low risk } \\ \mathbf{1}<\text { FPI }<\mathbf{1 0} & \text { Intermediate risk } \\ \mathbf{1 0}<\text { FPI }<\mathbf{1 0 0} & \text { High risk }\end{array}$

Hence, it is demonstrated that the simple addition of the micrometric outer membrane (50-60 micron) is able to reduce the fire propagating tendency of the epoxy-based composite, changing its classification from a high-risk material, to an intermediate one. These observations well compare with the behaviour highlighted by the FIGRA index, that has the same discussed trend and accounts for an overall sign that the coated composite is definitely less prone to propagating a fire.

It is worth to note that the overall better flame behaviour is achieved using a nanofibrous mat of only $24-27$ $\mathrm{g} / \mathrm{m}^{2}$ weight. The major improvement, as discussed above, concerns the Time To Ignition (TTI), which doubles. A similar improvement in TTI was also attained on polyurethane foams [26], but at the expense of the object lightness. In fact, four PMIA fabric layers $\left(155 \mathrm{~g} / \mathrm{m}^{2}\right.$ each) are required for such a result, leading to a significant weight increase. The object thickness is negatively affected too, since commercial PMIA fabrics with micrometric fiber diameter were used. PMIA nanofibrous mat guarantees the same TTI enhancement using less than a twentieth of flame retardant material weight $\left(24-27 \mathrm{~g} / \mathrm{m}^{2}\right.$ instead of $\left.620 \mathrm{~g} / \mathrm{m}^{2}\right)$. In the same work, it was demonstrated that the air permeability of PMIA layers plays a key role in the material fire behaviour. The higher the air permeability, the higher is the diffusion of inflammable gases evolved from the material surface. PMIA mats, being composed of nanofibers, should create a more efficient barrier effect against oxygen and inflammable gas diffusion than PMIA fabrics, leading to a more difficult combustion process. All the discussed results account for the polyaramidic nanofibrous coating as a smart way to modify the flame behaviour, even for objects which are already formed. The thinness and lightness $\left(24-27 \mathrm{~g} / \mathrm{m}^{2}\right)$ of the PMIA membrane do not significantly modify the object dimensions, and its pliability can make it to nicely adapt over complex surfaces. The fire-retardant behaviour that can be obtained with the use of such a simple approach appears, on the other side, significantly improved and it paves the way to a new approach in the fire retardancy field. In such a frame, however, the present work is put forward as a preliminary proof of concept 
for validating the idea, and still requires a systematic evaluation of the main factors influencing the performance, such the type of polymeric matrix effect (epoxy resins, vinyl ester resins, polyester resins, but also thermoplastics, such as polypropylene and polyphenylene sulfide), the membrane alignment (random or highly aligned) and thickness in order to fully understand the potential of PMIA nanofibers for the task at hand. Moreover, the fire response in different heat flux conditions, i.e. both representing the starting of the fire or its full development, should be also taken into account.

\section{CONCLUSIONS}

In the present work, the production of well defined polyaramidic nanofibrous membranes has been addressed, leading to some thin and well controlled Poly $(m$-phenylene isophtalamide) nanofibers with a significant alignment extent in the macroscopic mat. Such a membrane displays good mechanical properties and can be easily handled, so much that it could be applied onto epoxy based carbon fiber reinforce composites, with the aim of modifying their flame behaviour thanks to the intrinsic non-flammability of the PMIA. Conecalorimetric tests demonstrated that nanofiber covered composites display a delayed ignition, and they can be classified as intermediate risk materials in terms of ability of fire propagation, while the uncoated composite falls in the high risk rank. PMIA nanofibrous mat guarantees the same TTI enhancement of commercial fabrics using less than a twentieth of flame retardant material weight $\left(24-27 \mathrm{~g} / \mathrm{m}^{2}\right.$ instead of $\left.620 \mathrm{~g} / \mathrm{m}^{2}\right)$. All the discussed results account for the polyaramidic nanofibrous coating as a smart way to modify the flame behaviour, even for objects which are already formed, with significantly reduced hazard.

\section{Acknowledgements}

Funding: This work was supported by project TIME - Integrated Technology for Electric Mobility funded within POR FESR 2014-2020 action by Regione Emilia Romagna.

\section{Reference List}

[1] Hung P, Lau K, Cheng L, Leng J, Hui D. Impact response of hybrid carbon/glass fibre reinforced polymer composites designed for engineering applications. Composites Part B 2018;133:86-90.

[2] Chung DDL. Processing-structure-property relationships of continuous carbon fiber polymer-matrix composites. Mat Sci Eng R 2017;113(Supplement C):1-29.

[3] Mouritz AP, Feih S, Kandare E, Mathys Z, Gibson AG, Des Jardin PE, et al. Review of fire structural modelling of polymer composites. Composites Part A 2009;40(12):1800-14. 
[4] Price D, Anthony G, Carty P. World Fire Statistics Newsletter. The Geneva Association, editor. 29. 2014.

Ref Type: Case

[5] Mouritz A, Gibson AG. Flame Retardant Composites. In: Mouritz AP, Gibson AG, editors. Fire Properties of Polymer Composite Materials.Dordrecht: Springer Netherlands; 2006. p. 237-86.

[6] Zaikov GE, Lomakin SM. Ecological issue of polymer flame retardancy. J Appl Polym Sci 2002 Dec 5;86(10):2449-62.

[7] Stevens GC, Mann AH. Risks and benefits in the use of flame retardants in consumer products. Guilford: Report URN 98/1026 Great Britain Department of Trade and Industry; 1999.

[8] Jeencham R, Suppakarn N, Jarukumjorn K. Effect of flame retardants on flame retardant, mechanical, and thermal properties of sisal fiber/polypropylene composites. Composites Part B 2014;56(Supplement C):249-53.

[9] Kumar SK, Benicewicz BC, Vaia RA, Winey KI. 50th Anniversary Perspective: Are Polymer Nanocomposites Practical for Applications? Macromolecules 2017 Feb 14;50(3):714-31.

[10] Sisti L, Belcari J, Mazzocchetti L, Totaro G, Vannini M, Giorgini L, et al. Multicomponent reinforcing system for poly(butylene succinate): Composites containing poly(l-lactide) electrospun mats loaded with graphene. Polym Test 2016 Apr;50:283-91.

[11] Mazzocchetti L, Benelli T, D'Angelo E, Ligi S, Minak G, Poodts E, et al. Managing heat phenomena in epoxy composites production via graphenic derivatives: synthesis, properties and industrial production simulation of graphene and graphene oxide containing composites. 2D Mater 2017;4(1):015020.

[12] Zotti A, Borriello A, Ricciardi M, Antonucci V, Giordano M, Zarrelli M. Effects of sepiolite clay on degradation and fire behaviour of a bisphenol A-based epoxy. Composites Part B 2015;73(Supplement C):139-48.

[13] Benelli T, Mazzocchetti L, D'Angelo E, Lanzi M, Saraga F, Sambri L, et al. New nitrogen-rich heterocycles for organo-modified bentonites as flame retardant fillers in epoxy resin nanocomposites. Polym Eng Sci 2017 Jun 1;57(6):621-30.

[14] Zhang Y, Rhee KY, Park SJ. Nanodiamond nanocluster-decorated graphene oxide/epoxy nanocomposites with enhanced mechanical behavior and thermal stability. Composites Part B 2017;114:111-20.

[15] Liu S, Chevali VS, Xu Z, Hui D, Wang H. A review of extending performance of epoxy resins using carbon nanomaterials. Composites Part B 2018;136:197-214.

[16] Zhang Y, Choi JR, Park SJ. Thermal conductivity and thermo-physical properties of nanodiamondattached exfoliated hexagonal boron nitride/epoxy nanocomposites for microelectronics. Composites Part A 2017;101:227-36.

[17] Malucelli G, Carosio F, Alongi J, Fina A, Frache A, Camino G. Materials engineering for surfaceconfined flame retardancy. Mat Sci Eng R 2014;84:1-20. 
[18] Patrick Lim WK, Mariatti M, Chow WS, Mar KT. Effect of intumescent ammonium polyphosphate (APP) and melamine cyanurate (MC) on the properties of epoxy/glass fiber composites. Composites Part B 2012;43(2):124-8.

[19] Mouritz AP, Feih S, Kandare E, Gibson AG. Thermal-mechanical modelling of laminates with fire protection coating. Composites Part B 2013;48(Supplement C):68-78.

[20] Zhuge J, Gou J, Chen RH, Gordon A, Kapat J, Hart D, et al. Fire retardant evaluation of carbon nanofiber/graphite nanoplatelets nanopaper-based coating under different heat fluxes. Composites Part B 2012;43(8):3293-305.

[21] Wang W, Pan Y, Pan H, Yang W, Liew KM, Song L, et al. Synthesis and characterization of MnO2 nanosheets based multilayer coating and applications as a flame retardant for flexible polyurethane foam. Compos Sci Technol 2016;123:212-21.

[22] Zhang X, Shen Q, Zhang X, Pan H, Lu Y. Graphene oxide-filled multilayer coating to improve flameretardant and smoke suppression properties of flexible polyurethane foam. J Mater Sci 2016;51(23):10361-74.

[23] Mittal G, Rhee KY, Miškovic-Stankovic V, Hui D. Reinforcements in multi-scale polymer composites: Processing, properties, and applications. Composites Part B 2018;138:122-39.

[24] Gu H. Research on thermal properties of Nomex/Viscose FR fibre blended fabric. Materials \& Design 2009 Dec;30(10):4324-7.

[25] Du S, Wang W, Yan Y, Zhang J, Tian M, Zhang L, et al. A facile synthetic route to poly(p-phenylene terephthalamide) with dual functional groups. Chem Commun 2014;50(69):9929-31.

[26] Kotresh TM, Indushekar R, Subbulakshmi MS, Vijayalakshmi SN, Krishna Prasad AS, Gaurav K. Evaluation of foam/single and multiple layer Nomex fabric combinations in the cone calorimeter. Polym Test 2005;24(5):607-12.

[27] Levit NV, Young RH, inventors; Flame resistant thermal liner, composite fabric, and garment. US Patent patent US20160113340 A1. 2016 Apr 28.

[28] Merighi S, Maccaferri E, Belcari J, Zucchelli A, Benelli T, Giorgini L, et al. Interaction between Polyaramidic Electrospun Nanofibers and Epoxy Resin for Composite Materials Reinforcement. Key Eng Mat 2017;748:39-44.

[29] Armarego WLF, Perrin DD. Purification of Laboratory Chemicals. Oxford: Pergamon Press; 1966.

[30] Mazzocchetti L, D'Angelo E, Benelli T, Belcari J, Brugo TM, Zucchelli A, et al. Poly-m-aramid nanofiber mats: Production for application as structural modifiers in CFRP laminates. AIP Conf Proc 2016 May 18;1736(1):020016.

[31] Morgan AB, Wilkie CA. Flame Retardant Polymer Nanocomposites. Hoboken, NJ: John Wiley \& Sons, Inc.; 2007.

[32] Schartel B, Wilkie CA, Camino G. Recommendations on the scientific approach to polymer flame retardancy: Part 1ГÇöScientific terms and methods. J Fire Sci 2016 Nov 1;34(6):447-67.

[33] Yao L, Lee C, Kim J. Fabrication of electrospun meta-aramid nanofibers in different solvent systems. 
Fibers Polym 2010;11(7):1032-40.

[34] Ryu SY, Chung JW, Kwak SY. Amphiphobic meta-aramid nanofiber mat with improved chemical stability and mechanical properties. Eur Polym J 2017;91:111-20.

[35] Schartel B, Hull TR. Development of fire-retarded materialsГÇöInterpretation of cone calorimeter data. Fire Mater 2007 Aug 1;31(5):327-54.

[36] Petrella RV. The Assessment of Full-Scale Fire Hazards from Cone Calorimeter Data. J Fire Sci 1994 Jan 1;12(1):14-43. 
Table 1: composition and processing parameters of PMIA electrospun solutions

\begin{tabular}{ccccccccc}
\hline Solution & $\begin{array}{c}\text { PMIA } \\
(\% \mathbf{w t} / \mathbf{w t})\end{array}$ & $\begin{array}{c}\text { LiCl } \\
(\boldsymbol{\%} \mathbf{w t} / \mathbf{w t})\end{array}$ & $\begin{array}{c}\text { DMAc } \\
(\boldsymbol{\%} \mathbf{w} / \mathbf{w t})\end{array}$ & $\begin{array}{c}\text { Collector } \\
\mathbf{d i s t a n c e} \\
(\mathbf{c m})\end{array}$ & $\begin{array}{c}\text { Flow } \\
\text { Rate } \\
(\mathbf{m l} / \mathbf{h})\end{array}$ & $\begin{array}{c}\text { Voltage } \\
(\mathbf{k V})\end{array}$ & $\begin{array}{c}\text { HR } \\
(\boldsymbol{\%})\end{array}$ & $\begin{array}{c}\mathbf{T} \\
\left(\mathbf{C}^{\circ}\right)\end{array}$ \\
\hline PMIA 14-2 & 14 & 2 & 84 & 15 & 0.4 & 16 & 45 & 21.8 \\
\hline PMIA 14-2,5 & 14 & 2.5 & 83.5 & 15 & 0.2 & 17.2 & 25 & 27 \\
\hline PMIA 14-3,5 & 14 & 3.5 & 82.5 & 15 & 0.1 & 25 & 27 & 23.6 \\
\hline
\end{tabular}

Table

data of

Sample

$\Delta \mathbf{H}(\mathbf{J} / \mathbf{g})^{\mathbf{a}}$ 208

PMIA,

$\begin{array}{cc}\text { Neat PMIA } & 208 \\ \text { PMIA W } & 222 \\ \text { PMIA UW } & 637\end{array}$

\section{$\mathbf{T}_{\text {endothermic peak }}\left({ }^{\circ} \mathbf{C}\right)^{\mathbf{a}}$} $\mathbf{T}_{\mathrm{g}}\left({ }^{\circ} \mathbf{C}\right)^{\mathrm{b}}$

2: $\quad$ DSC

and PMIAUW

${ }^{\text {a }}$ First DSC scan endothermic transition

${ }^{\mathrm{b}}$ Second DSC scan

Table 3: Mechanical properties of PMIA nanofibrous mats

\begin{tabular}{|c|c|c|c|}
\hline Sample & $\mathbf{E}(\mathbf{M P a})$ & $\mathbf{I}_{\mathrm{a}}^{\mathrm{a}}$ & $\sigma_{\mathrm{r}}(\mathrm{MPa})$ \\
\hline PMIA 14-3,5 W // & $679 \pm 77$ & \multirow{2}{*}{3.95} & $27 \pm 2$ \\
\hline PMIA 14-3,5 W $\perp$ & $172 \pm 13$ & & $5 \pm 0.1$ \\
\hline PMIA 14-2 W // & $752 \pm 71$ & \multirow{2}{*}{6.96} & $30 \pm 2$ \\
\hline PMIA 14-2 W $\perp$ & $108 \pm 6$ & & $3 \pm 0.1$ \\
\hline PMIA 14-2 UW // & 170 & I & 3 \\
\hline
\end{tabular}

a) Alignment Index (Ia) described in eq. 1 .

Table 4: Cone Calorimeter parameters

\begin{tabular}{ccccc}
\hline Sample & C-Neat & C-PMIA inc W & CP-Neat & CP-PMIA W \\
\hline HRR $\left(\mathbf{k W} / \mathbf{m}^{\mathbf{2}}\right)$ & $86 \pm 7$ & $85 \pm 4$ & $66 \pm 1$ & $57 \pm 12$ \\
\hline pHRR $\left(\mathbf{k W} / \mathbf{m}^{\mathbf{2}}\right)$ & $478 \pm 53$ & $382 \pm 17$ & $470 \pm 50$ & $391 \pm 30$ \\
\hline THR $\left(\mathbf{M J} / \mathbf{m}^{\mathbf{2}}\right)$ & $22.4 \pm 0.2$ & $19.8 \pm 1.0$ & $18.8 \pm 0.4$ & $19.7 \pm 0.9$ \\
\hline TTI $(\mathbf{s})$ & $53 \pm 1$ & $52 \pm 1$ & $46 \pm 1$ & $94 \pm 15$ \\
\hline TTP $(\mathbf{s})$ & $68 \pm 6$ & $81 \pm 1$ & $76 \pm 16$ & $122 \pm 20$ \\
\hline TOF $(\mathbf{s})$ & $193 \pm 9$ & $185 \pm 29$ & $193 \pm 10$ & $240 \pm 6$ \\
\hline FPI $\left(\mathbf{k W} / \mathbf{m}^{\mathbf{2}} \mathbf{s}\right)$ & $9.0 \pm 0.8$ & $7.4 \pm 0.3$ & $10.2 \pm 1.4$ & $4.3 \pm 1.0$ \\
\hline FIGRA $\left(\mathbf{k W} / \mathbf{m}^{\mathbf{2}} \mathbf{s}\right)$ & $7.1 \pm 1.2$ & $4.7 \pm 0.2$ & $6.4 \pm 2.0$ & $3.3 \pm 0.8$ \\
\hline
\end{tabular}




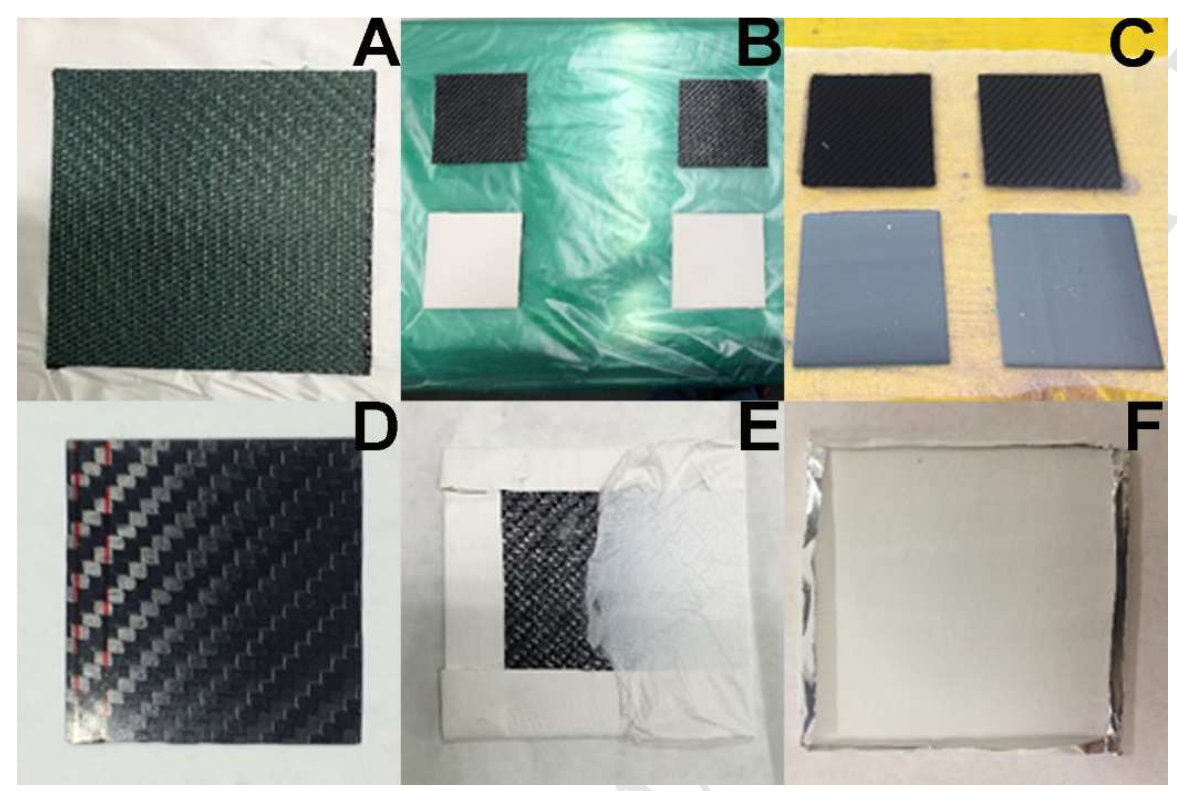

Figure 1. C-Neat sample (A), C-PMIA inc W sample (B and C), CP-Neat sample (D) and CP-PMIA W sample $(\mathrm{E}$ and $\mathrm{F})$ at different stages of the covering procedure.

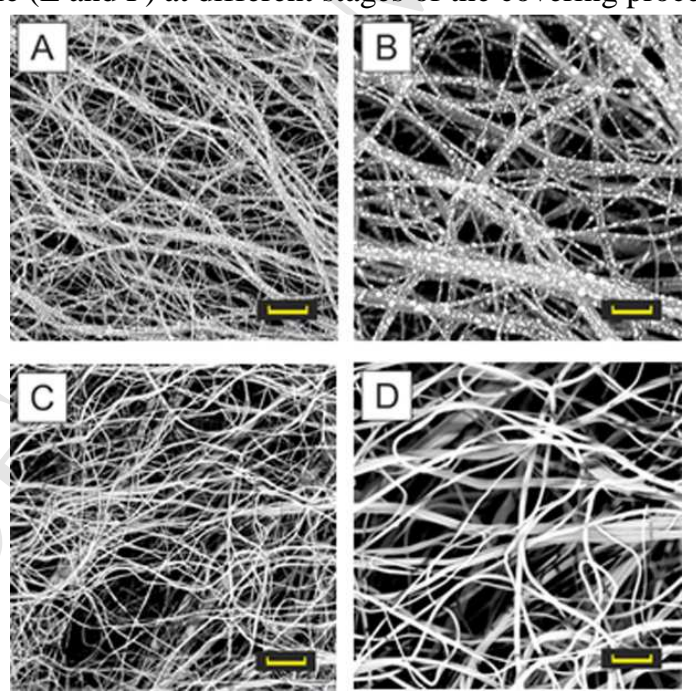

Figure 2 - SEM micrographs of PMIA UW (A - 6000X, B - 15000X) and PMIA W (C - 6000X, D 15000X) electrospun mats. Scale bar: $5 \mu \mathrm{m}$ 6000X, $2 \mu \mathrm{m}$ 15000X micrographs. 

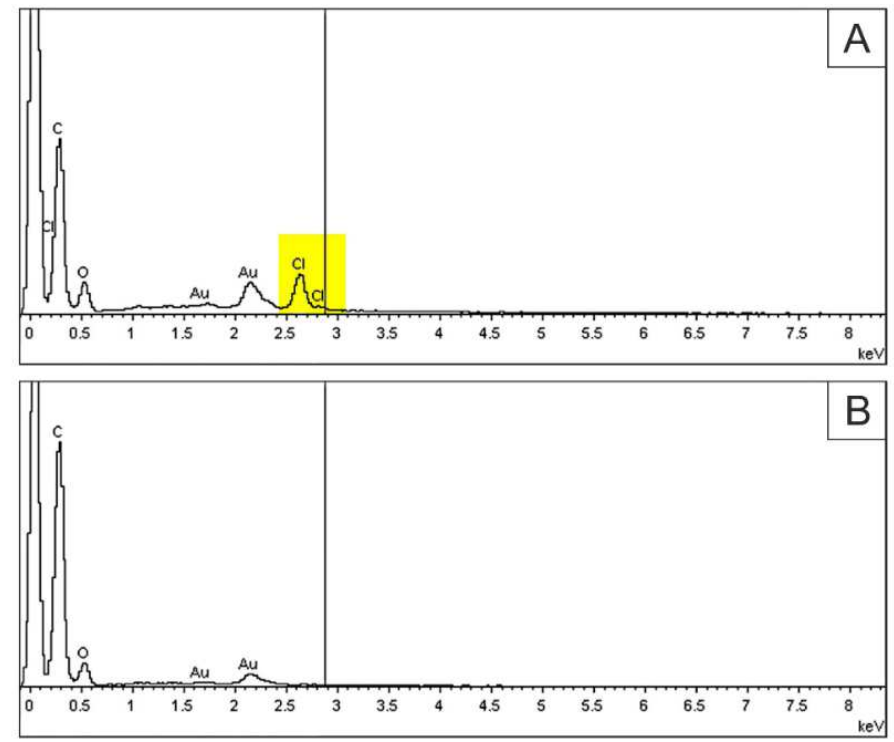

Figure 3 - EDX spectra of PMIA UW (A) and PMIA W (B) mats.

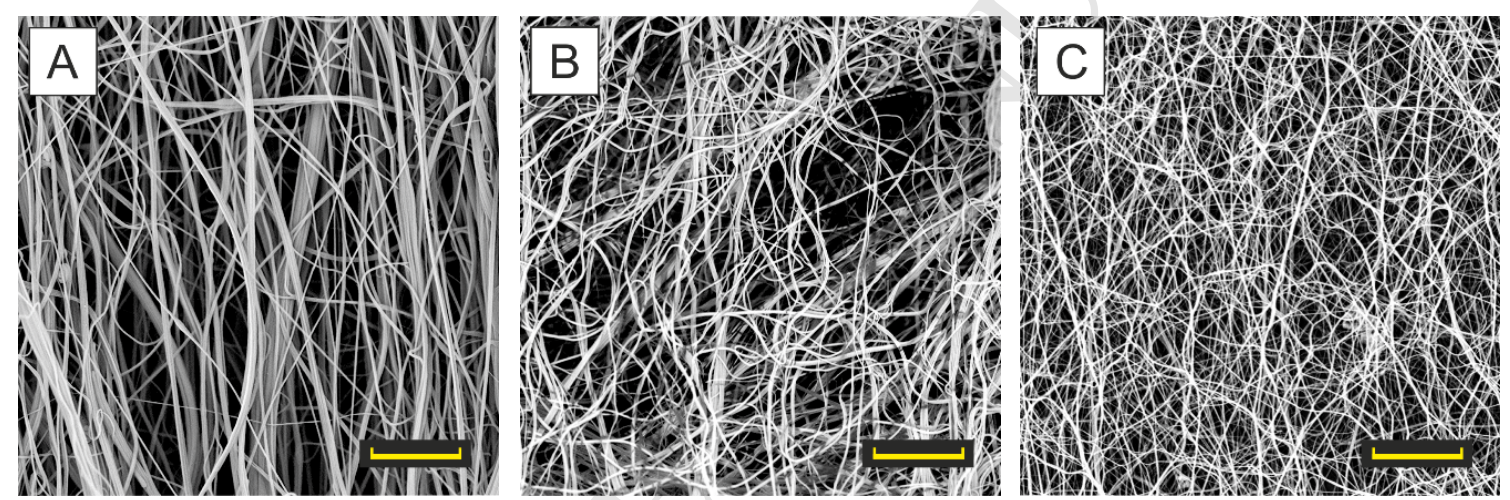

Figure 4 - SEM micrographs of washed PMIA with 14\% wt/wt of PMIA and 2 (A)-2,5 (B)-3,5\% (C) of LiCl $(\mathrm{A}-5000 \mathrm{X}, \mathrm{B}-5000 \mathrm{X}, \mathrm{C}-5000 \mathrm{X})$ Scale bar: $10 \mu \mathrm{m}$ 5000X

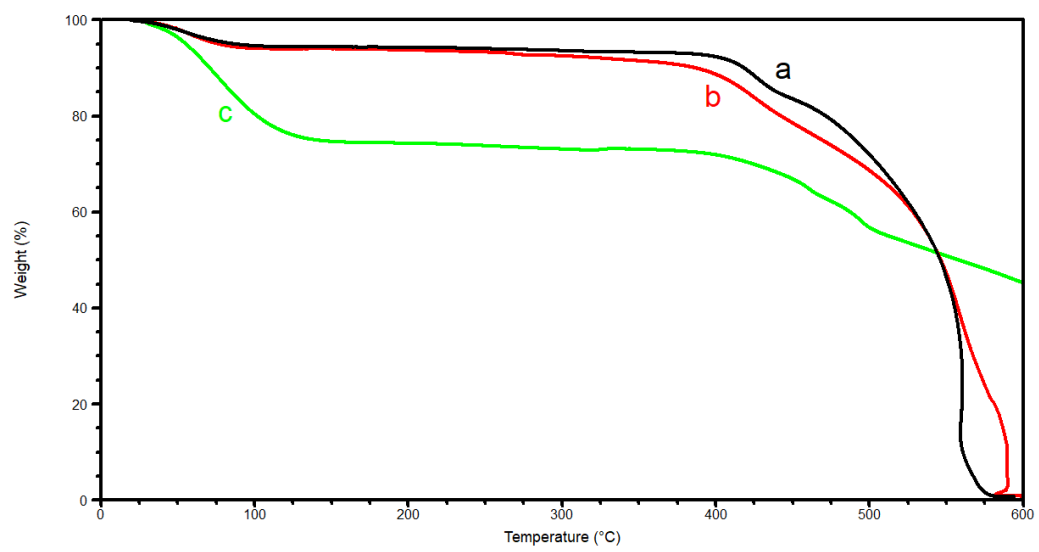

Figure 5. TGA curves in air of (a) neat PMIA, (b) PMIA W and (c) PMIA UW nanofibrous membranes. 

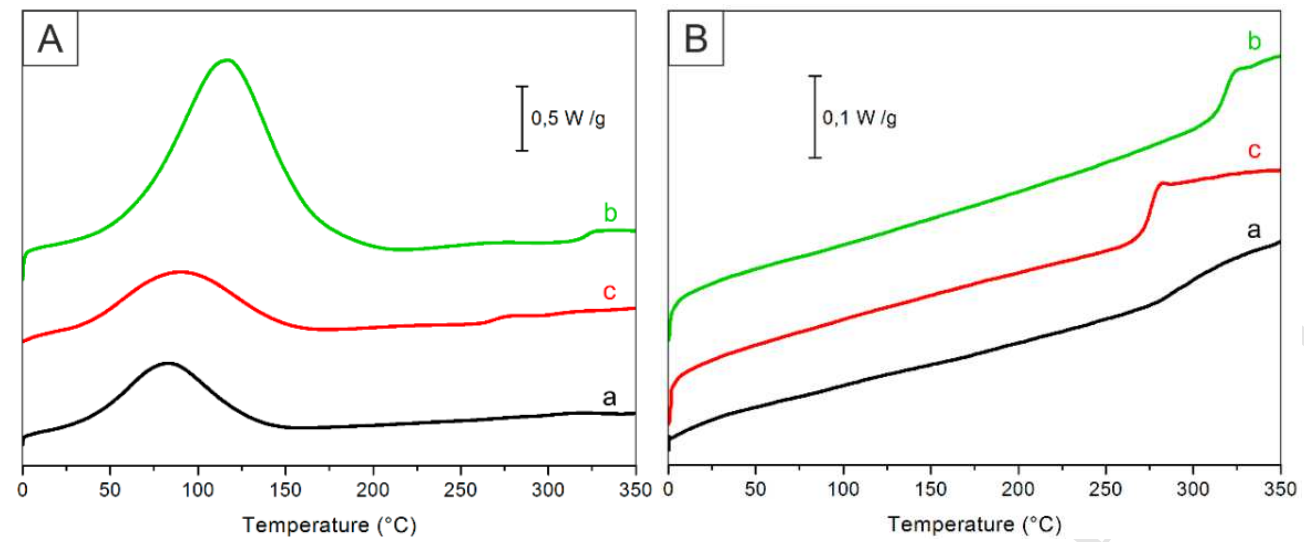

Figure 6. First (A) and second scan (B) DSC termograms of (a) neat PMIA, (b) PMIA UW, and (c) PMIA W.
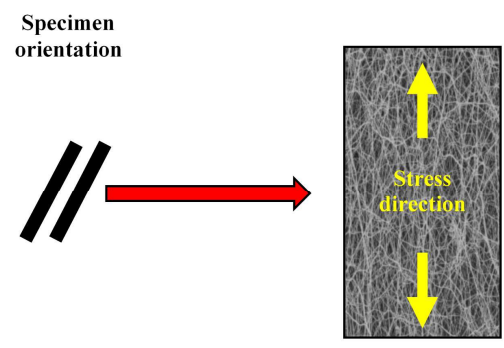

Drum rotation axis
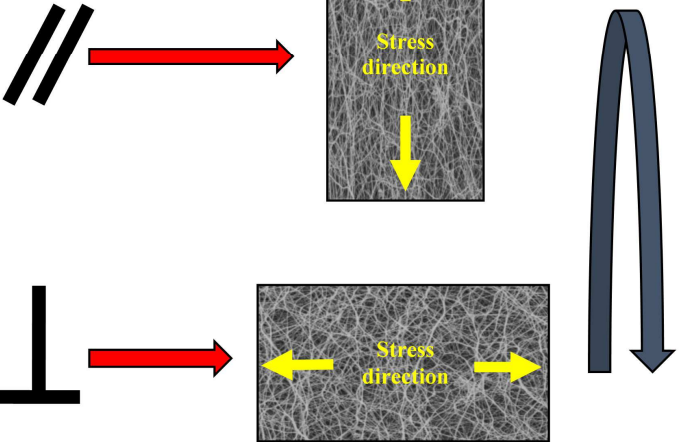

Figure 7. PMIA mat samples collected along the direction corresponding either to the longitudinal (//) or the transverse $(\perp)$ direction of the rotating drum collector. The nanofibrous samples are prevailing aligned along the drum rotation axis.
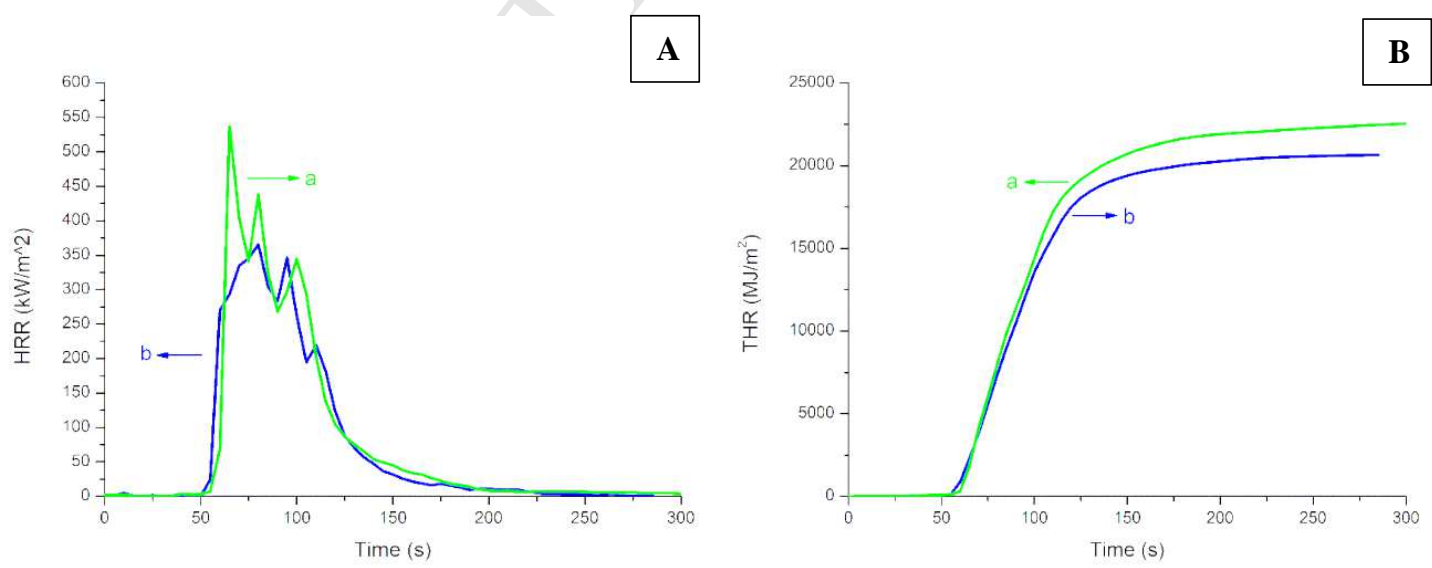

Figure 8: Comparison of two significative cono calorimetric curves reporting HRR (A) and THR (B) behaviour of: (a) C-Neat (green) and (b) C-PMIA inc W (blue). 

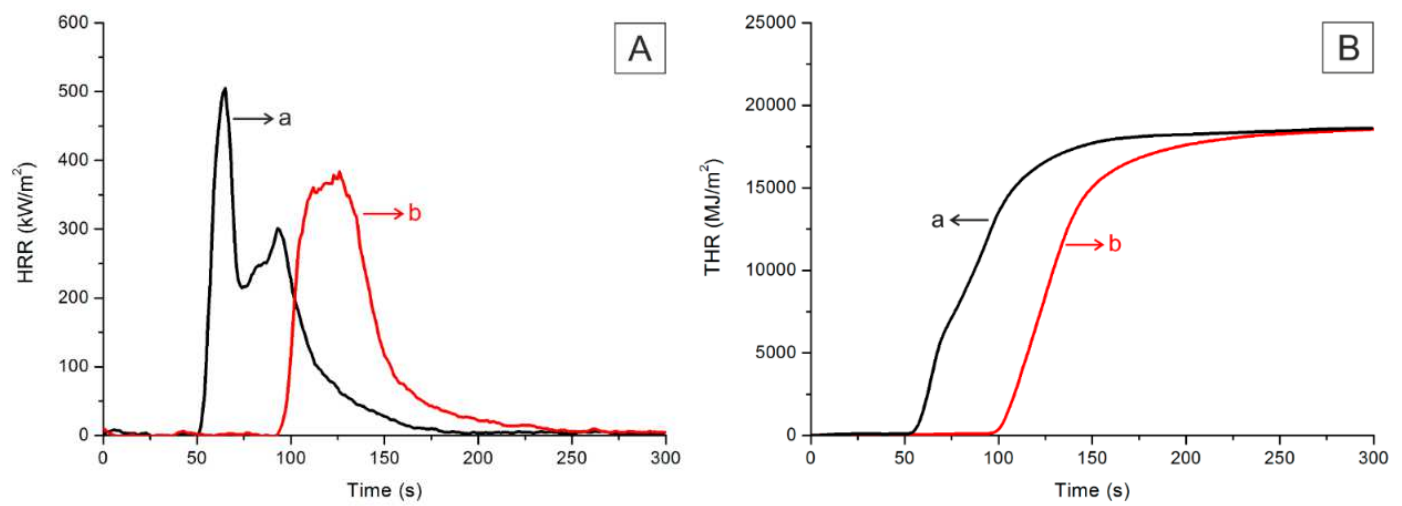

Figure 9: Comparison of two significative cone-calorimetric curves reporting HRR (A) and THR (B) behaviour of: (a) CP Neat (black) and (b) CP PMIA W (red).

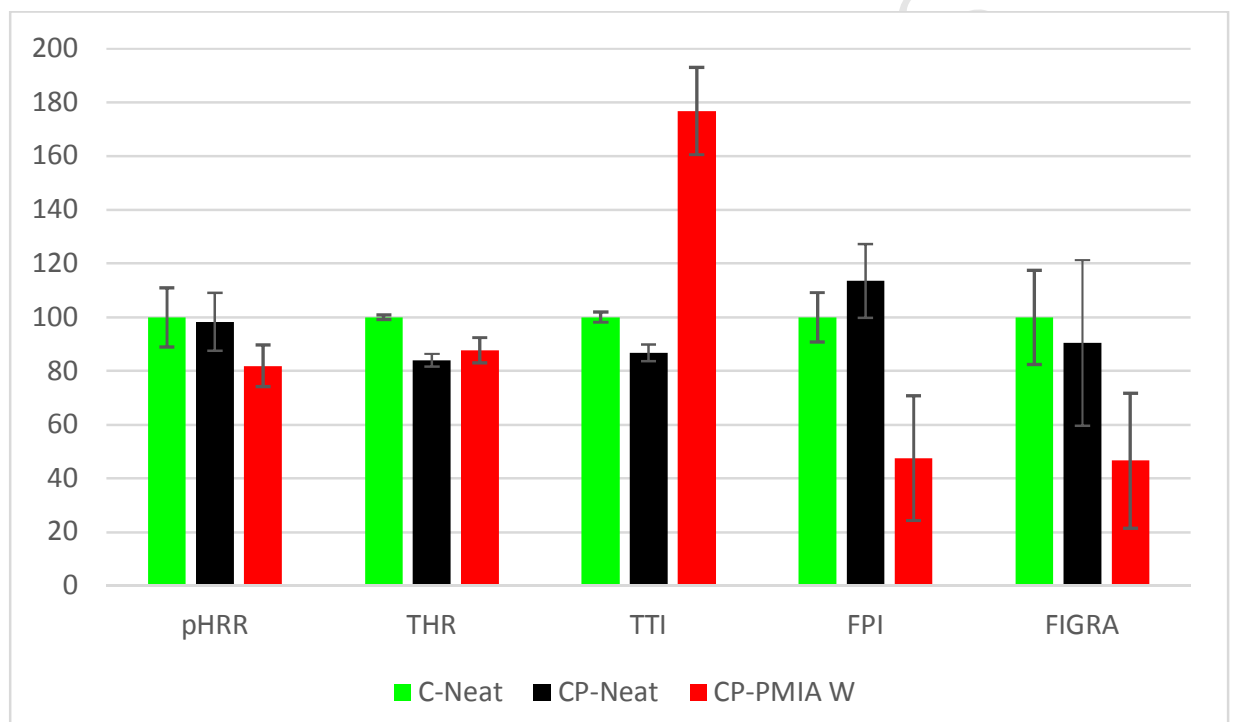

Figure 10: Fire behaviour significant data, pHRR, THR, TTI, FPI and FIGRA, relative to C-Neat (green), CP Neat(black) and CP PMIA W (red), expressed as \% variation with respect to the plain neat composite CP Neat, with the relative standard deviation. 